\title{
Simulation of Signal Processing Scheme for Fiber Optic Rotationary Sensor
}

\author{
G. Siva Sravani, K.Gouthami, K. Sai Mounika, J.Chandra Sekhar
}

\begin{abstract}
Fiber optic revolving sensor is a significant improvement in the field of inertial sensors and it is presently considered as an elective innovation to mechanical and ring laser whirligigs for the inertial route and control applications. Fiber optic turn sensor is chipping away at the standard of Sagnac impact, which expresses that an optical way distinction incited by counter spreading bars in a pivoting reference outline is corresponding to the outright revolution. The sensors output is a cosine function of rotation rate. The rotation rate extraction scheme deals with problems of power variations of source and ambiguity at directions. Fiber optic rotation sensors can be classified into two different types
\end{abstract}

$>\quad$ Open-loop fiber-optic rotation sensors

$>\quad$ Closed-loop fiber optic rotation sensors

When considered both the types, closed loop fiber-optic rotation sensors are generally more stable than open loop fiberoptic rotation sensors. Even closed loop fiber optic sensors can be implemented in a simpler way compared to the open loop fiber optic rotation sensor. So in order to overcome these problems, a modification of the harmonic division algorithm is to be developed with which open loop fiber optic rotation sensor is made more stable and likewise less difficult for execution than the first one. At the point when the simple sign is appropriately examined and quantized, the presentation of the calculation achieves that of fiber optic revolving sensors and the usage of this calculation should be possible by utilizing off-the-rack part, and henceforth does not require a coordinated optics circuit.

Index Terms - open loop closed loop, rotation sensor, fiber optics sensors

\section{INTRODUCTION}

The main Fiber Optic Rotation Sensor was shown in the US by Vali and Shorthill in 1976. Advancement of both the latent interferometer sort of FOS and the aloof ring resonator kind of FOS is continuing in numerous organizations and foundations around the world. An optical gyrator, laser or fiber, measures the impedance design created by two light bars, going in inverse headings inside a reflected ring or fiber circle, so as to recognize little changes in movement.

Fiber-Optic Rotation Sensor can work with two distinct setups:

$>$ Open loop

$>$ Closed loop

Open loop fiber optic rotation sensor

Revised Manuscript Received on April 12, 2019.

G.SivaSravani, Department of ECE, MalineniLakshmaiah women's Engineering College, Guntur.A.P, India

Dr.K.Gouthami, Department of ECE,MalineniLakshmaiah women's Engineering College, Guntur.A.P, India

K.SaiMounika, Department of ECE, MalineniLakshmaiah women's Engineering College, Guntur.A.P, India

J.ChandraSekhar, Department of ECE, KL University, Vaddeswaram. A.P, India
In the open circle design the data about the angular rate is acquired straightforwardly by the electrical sign at the yield. In the shut circle a non -equal stage move is produced in the criticism circle to invalid the all out stage move. The connected inverse stage move gives the data about the revolution rate.

As in any interferometric gadget, the yield signal is the consequence of the obstruction between two waves that, for this situation, are counter proliferating in the optical fiber To precisely quantify the Sagnac impact and, at that point, increment the gyrator quality, it is important to lessen any stage contrasts not quite the same as that prompted by the turn

The block diagram of open-loop fiber optic rotation sensor as shown below

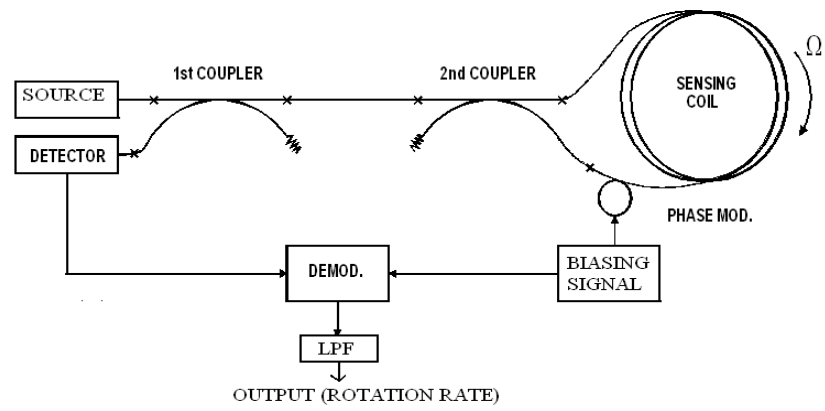

Figure I-1. Open Loop Fiber Optic Rotation Sensor

The open loop fiber optic rotationary sensor is an interferometer where a light source i.e., Super Luminescent Diode (SLD) is divided in to two beams, which propagate along clock wise and counter clock wise paths in optical fiber sensor coil and then recombined before being detected by a PIN photodiode based fiber optical receiver. In the presence of rotation, the two beams from the normal source experience distinctive stage moves before recombining on the optical indicator.

The stage modulator is utilized to apply a dynamic stage inclination created by a sinusoidal wave to the light way, in this manner expanding affectability. On the off chance that the balance recurrence $\mathrm{fm}$ is $\mathrm{s}$ adequately high, the electronic clamor is maintained a strategic distance from. The sign at the yield of the photodiode is demodulated and prepared for delivering a yield relating to the greatness and feeling of the pivot.

In the plan the demodulator yield goes through a servo enhancer which drives a stage transducer put in the interferometer. The absolute stage move ends up equivalent

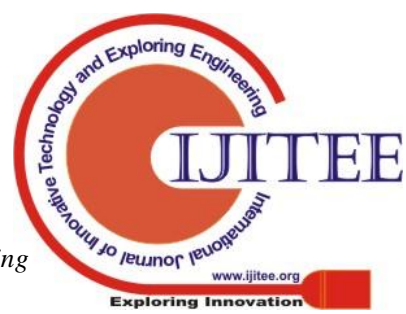


to zero on the grounds that the stage transducer presents a non-equal stage move that is equivalent, however inverse in sign, to that created by the Sagnac impact when the gadget pivots. The yield of the framework is then the yield of the stage transducer

\section{Closed-loop Fiber Optic Rotation sensor}

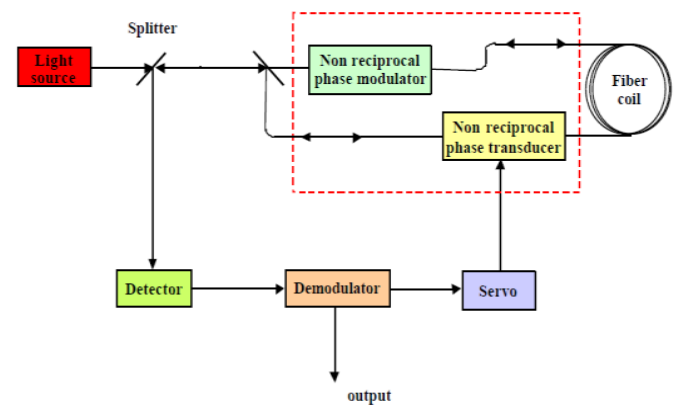

Figure2. Block Diagram of Closed Loop Fiber Optic Rotational Sensor

With the advancement of optical filaments, analysts started to investigate various methods for utilizing this innovation to make optical pivot sensors, which had the option to coordinate or surpass the exactness of contemporary mechanical sorts. The most significant preferred position was the absence of the moving parts, which upgrades unwavering quality and exactness.

The FOS commonly demonstrates higher goals than a ring laser spinner however used to have less steady inclination and more awful scale factor execution until the finish of the 90's. Since the start of the century, High Performance FOS has exhibited preferable inclination steadiness over the most precise ring laser gyrators. Rotation Sensor

The main limitations of this basic configuration are

$>$ Poor sensitivity for small rotation rates.

$>$ The direction of rotation cannot be determined

$>$ The output is a nonlinear function of rotation rate.

\section{IMPLEMENTATION OF SIGNAL PROCESSING SCHEME FOR FIBER OPTIC ROTATIONARY SENSOR}

\subsection{Principle of Fiber Optic Rotation Sensor}

Sagnac first demonstrated the optical rotation sensor principle in 1913, fiber optic rotation sensors are implemented by using this principle.

\subsection{Sagnac Effect}

It expresses that an optical way distinction prompted by counter proliferating bars in a turning reference outline is corresponding to the outright revolution. The initiated optical way contrast can be estimated in two waves

a) By making a frequency measurement in a laser resonator or in a fiber optic rotation sensor.

b) By a phase measurement of two interfering beams as in an Interferometer Fiber Optic Gyroscope (IFOG).

In spite of the fact that as far as hypothetical execution the two frameworks began with similar desires. By and by IFOG based inertial measurement unit (IMU) has been flight tried and is being delivered by numerous ventures far and wide, where as RFOG is still in research center stages.

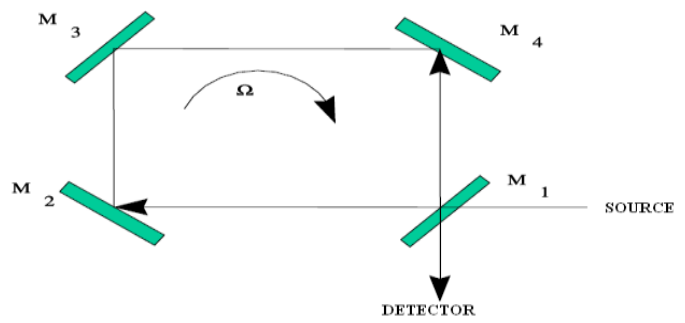

Figure3. Sagnac Interferometer Bulk Optics

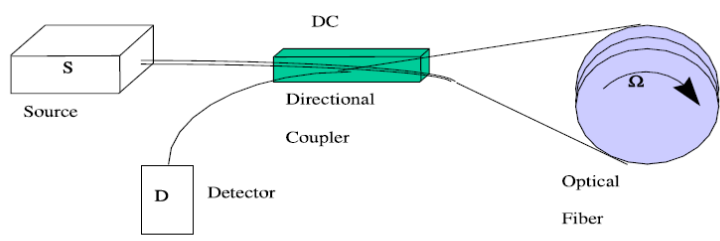

Figure 4. Fiber Optics

When system is at rest the light propagates in clockwise (CW) and counter clockwise (CCW) directions in transverse identical paths and so there is no phase difference between them. When the system rotates at an angular velocity $\Omega$ then CW path will be longer by (LR / C) $\Omega$ and CCW path will be shorter by $(\mathrm{LR} / \mathrm{C}) \Omega$ i.e

$$
\begin{aligned}
\mathrm{L}_{\mathrm{CCW}} & =2 \pi \mathrm{R}-\mathrm{R} \Omega \mathrm{t}_{\mathrm{CCW}} \\
\mathrm{L}_{\mathrm{CW}} & =2 \pi \mathrm{R}+\mathrm{R} \Omega \mathrm{t}_{\mathrm{CCW}}
\end{aligned}
$$

This yield,

$\Delta \mathrm{L}=\mathrm{C}\left(\mathrm{t}_{\mathrm{CW}}-\mathrm{t}_{\mathrm{CCW}}\right) 4 \mathrm{~A} \Omega / \mathrm{C}$

Thus the phase difference produced between $\mathrm{CW}$ and $\mathrm{CCW}$ wave is obtained

$\Delta \phi_{R}=\frac{4 \pi R L}{\lambda C} \Omega$

Equation (1)

$\Delta \phi_{\mathrm{R}}=$ Phase shift due to rotation rate,

$R=$ Radius of path,

$L=$ Length of the coil and $\mathrm{C}$ is the speed of light,

$\lambda=$ Source wavelength

\subsection{Sensor Design}

\subsubsection{Wavelength selection}

For the same coil dimension and the same returning power, the Sagnac phase difference is inversely proportional to the wavelength and the signal - to - noise ratio is proportional to the square root of the wavelength, since, as the wavelength increases, the photon energy decreases, increasing their number for a given power. Any of the usual transparency windows (i.e., 850, 1060, 1300, 1550nm) of silica fibers have been used with little difference in performance. The graphical representations of optical losses at different wavelengths are shown below 


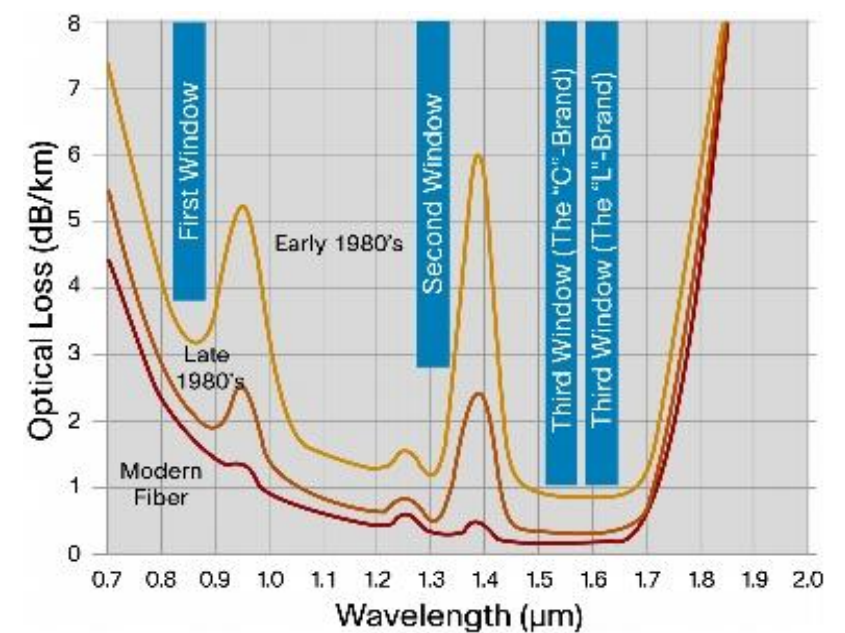

Graph1. Representation of Wave Length versus Optical Loss

The advantage of the very low attenuation of the 1330 or 1550-nm windows. The criteria of choice are mainly economic and based on cost, availability, and standardization of the components, unless the gyro has to withstand radiation, which makes the long wavelength the range necessary to avoid any increase of the fiber attenuation. Therefore wavelength $1550 \mathrm{~nm}$ is selected in the development of FOG.

\subsubsection{Sensitivity}

As in any passive optical system, the theoretical sensitivity of the FOS is limited by the photon shot noise. Assuming a phase bias of $\pi / 2 \mathrm{rad}$ to operate the interferometer at the inflection point of the cosine response for maximum sensitivity, the detector power is

$$
I=I_{0}\left[1+\cos \left(\Delta \phi_{R}+\pi / 2\right)\right]
$$

However, an optical beam may be regarded as a stream of photons, which behaves statically like any ensemble of uncorrelated discrete particles. Any flow $N=d N / d t$ yields a random counting with a standards deviation $\sigma \mathrm{N}$ of $\mathrm{N}$ following

$$
\sigma_{N}^{2}=2 N \Delta f_{b w}
$$

Where, $\Delta \mathrm{fbw}$ is the counting bandwidth, for photons with an energy $h f=h e / \lambda$ (where $h=6.63 \times 10-34$ ) Js is the Plank constant). The optical power $\mathrm{P}$ and its standard deviation $\sigma \mathrm{p}$ follow

$$
\begin{aligned}
& {\left[\frac{\sigma P}{h e / \lambda}\right]^{2}=2 \frac{P}{h e / \lambda} \Delta f_{b w}} \\
& \sigma P / \sqrt{\Delta f_{b w}}=\sqrt{2 .(h c / \lambda) .}
\end{aligned}
$$

For $\lambda=1550 \mathrm{~nm}$

$$
\sigma P / \sqrt{\Delta f_{b w}}=0.7 \times 10^{-9} \sqrt{P}(\mathrm{P} \text { in watts })
$$

\section{SIMULATION RESULTS}

\subsection{Source Code}

The above algorithm has been simulated and implemented using MATLAB tool.

clc

clear all

close all

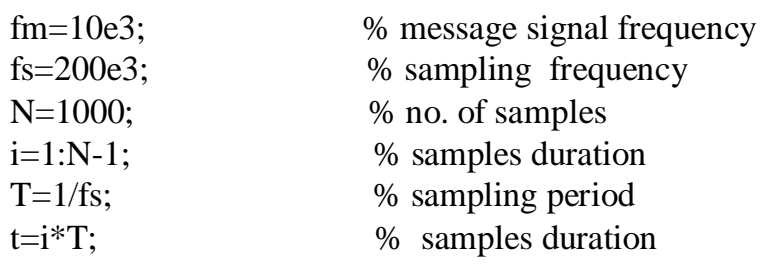

$\mathrm{fm}=10 \mathrm{e} 3$

$\mathrm{fs}=200 \mathrm{e} 3$;

$\mathrm{N}=1000$;

$\mathrm{i}=1: \mathrm{N}-1$;

$\mathrm{T}=1 / \mathrm{fs}$;

$\mathrm{t}=\mathrm{i}^{*} \mathrm{~T}$

$\%$ message signal frequency

$\%$ sampling frequency

$\%$ no. of samples

$\%$ samples duration

$\%$ sampling period

$\%$ samples duration

$\mathrm{x}=1$;

$\Delta \phi_{\mathrm{R}}=0$

$\Delta \phi_{\mathrm{m}}=\mathrm{pi} / 2$

$\mathrm{wm}=2 * \mathrm{pi}^{*} \mathrm{fm} * \mathrm{t}$;

$\mathrm{q}=\sin (\mathrm{wm}) ; \quad \%$ sine signal

subplot $(5,1,1)$

$\operatorname{plot}(\mathrm{q})$

$\mathrm{d}=\Delta \phi_{m} * \mathrm{q}$;

$\mathrm{R}=\Delta \phi_{\mathrm{R}}+\mathrm{d}$

$\mathrm{E}=\cos (\mathrm{R})$;

$\mathrm{I}=\mathrm{I}_{0} *(1+\mathrm{E})$;

configuration is

subplot $(5,1,2)$

plot(I)

$\mathrm{c}=\mathrm{fft}(\mathrm{I}) ; \quad \%$ fourier transform of detector output

subplot $(5,1,3)$

plot $(\operatorname{abs}(\mathrm{c})) \quad \%$ abs=complex conjugate

$\mathrm{z}=\mathrm{abs}(\mathrm{c})$

$\mathrm{f} 1=\mathrm{i} / \mathrm{N} ; \quad \%$ 0-1 digital values

$\mathrm{f} 2=\mathrm{f} 1 * \mathrm{fs} ; \quad \%$ signal converted to frequency range

$\mathrm{N} 2=\operatorname{round}(\mathrm{N} / 2) ; \quad \%$ rounding the decimal values to

integer values

subplot $(5,1,4)$

$\operatorname{plot}(\mathrm{f}(1: \mathrm{N} 2), \mathrm{I}(1: \mathrm{N} 2))$;

grid on

subplot $(5,1,5)$

plot(f(1:N2),z(1:N2));

grid on

$\mathrm{D} 1=\mathrm{I}^{*} \sin (\mathrm{wm})$;

$\mathrm{D} 2=\mathrm{I} * \cos (2 * \mathrm{wm})$;

$\mathrm{D} 3=\mathrm{I} * \sin (3 * \mathrm{wm})$;

$\mathrm{D} 4=\mathrm{I}^{*} \cos (4 * \mathrm{wm})$

$\mathrm{Z}=$ lpfcoeff;

$\mathrm{S} 1=\operatorname{conv}(\mathrm{D} 1, \mathrm{Z})$;

figure()

plot(S1)

$\mathrm{S} 2=\operatorname{conv}(\mathrm{D} 2, \mathrm{Z})$;

figure()

plot(S2)

$\mathrm{S} 3=\operatorname{conv}(\mathrm{D} 3, \mathrm{Z})$;

figure()

plot(S3)

S4=conv(D4,Z);

figure()

plot(S4)

$\mathrm{P} 1=\mathrm{S} 1 / \mathrm{S} 2$;

figure()

plot(P1)

$\mathrm{P} 2=\mathrm{S} 2 / \mathrm{S} 4$;

figure()

$\operatorname{plot}(\mathrm{P} 2)$

$\%$ detector output when basic 
On changing the values of $A$ that is the rotational rate the results obtained will be as shown in the following figures.

\subsection{Test Results :}

On changing the values of a the output changes accordingly
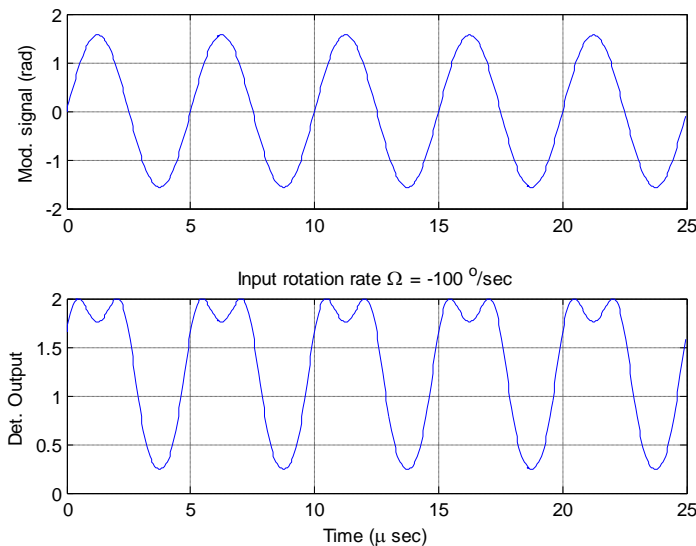

Graph1. Graphical Representation of Detector O/P For Rotation Rate of $-100 \mathrm{deg} / \mathrm{Sec}$

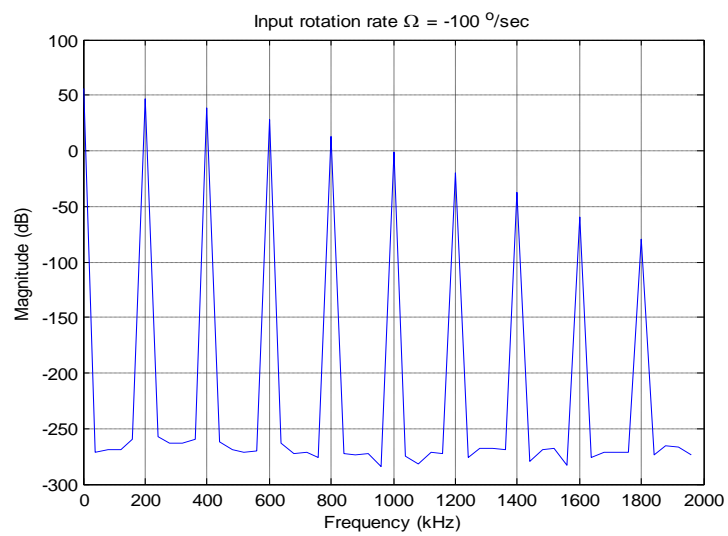

Graph 1: Graphical representation of detector o/p fft for rotations rate of $-100 \mathrm{deg} / \mathrm{sec}$
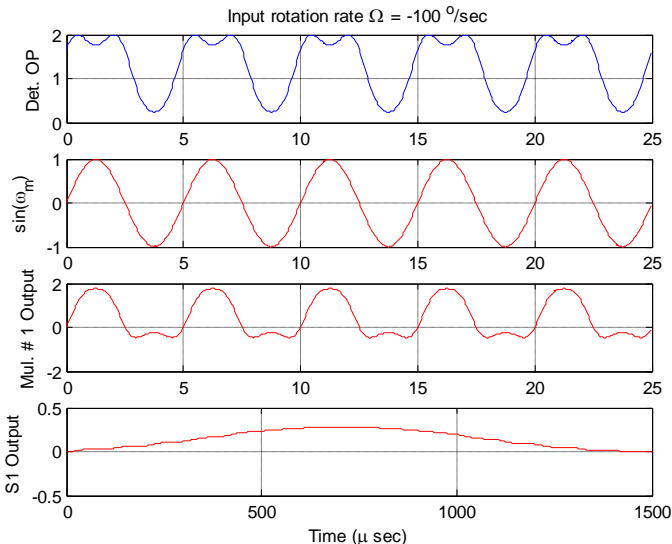

Graph 3. Graphical representation low pass filtered S4 for rotation rate of $100 \mathrm{deg} / \mathrm{sec}$
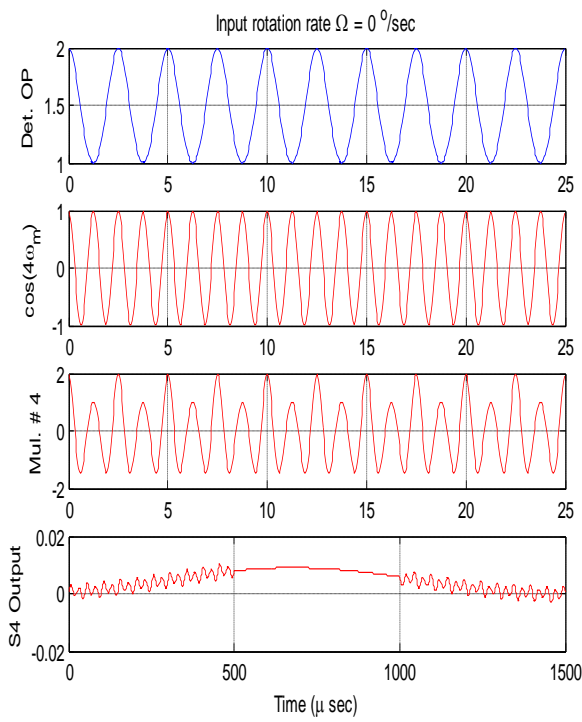

Graph4. Graphical representation of detector $\mathbf{o} / \mathrm{p}$ for rotation rate of $0 \mathrm{deg} / \mathrm{sec}$.
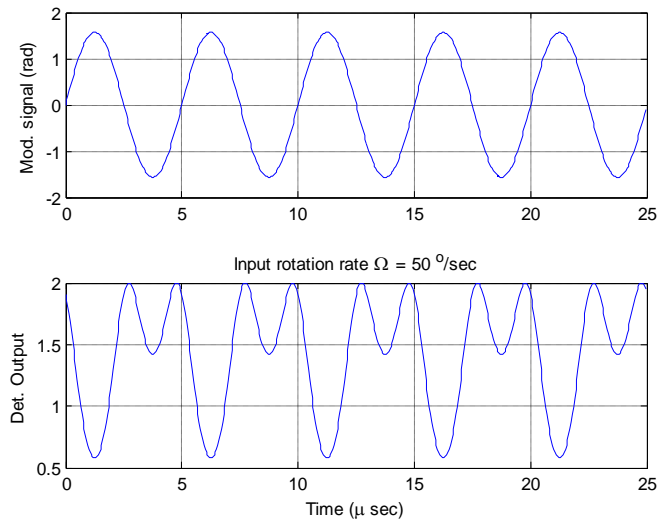

Graph 5. Graphical representation of fft for detector o/p for rotation rate of $50 \mathrm{deg} / \mathrm{sec}$
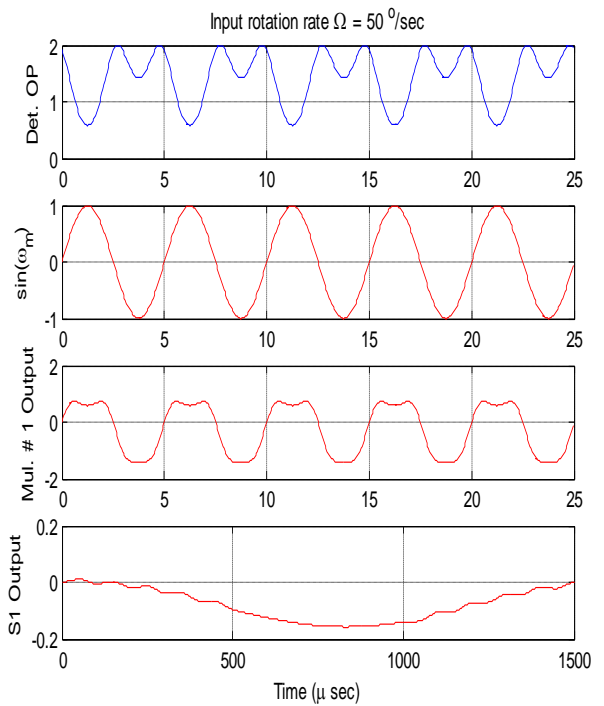

Graph III. Graphical representation low pass filtered S1 for rotation rate of $50 \mathrm{deg} / \mathrm{sec}$ 

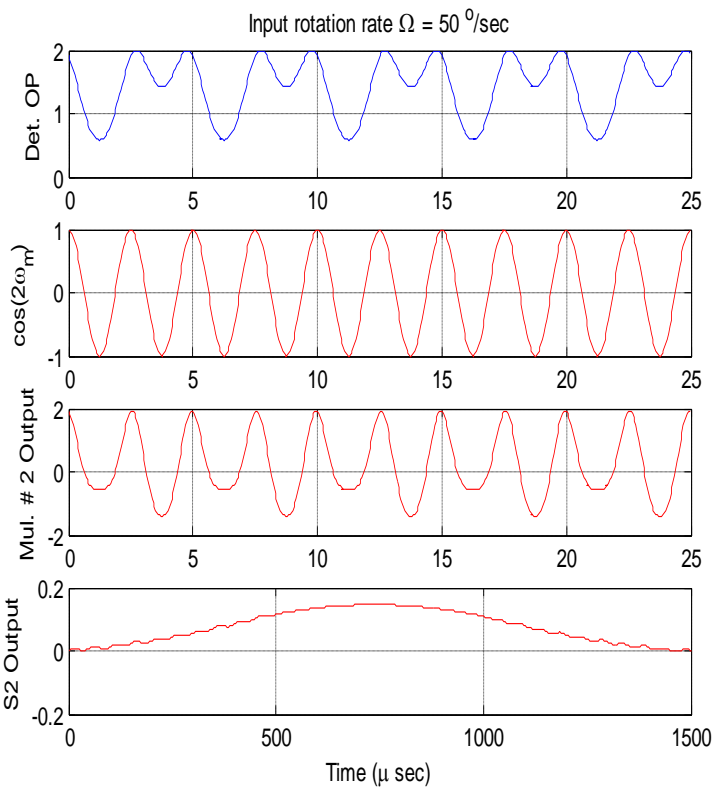

Graph .7 Graphical representation low pass filtered S2 for rotation rate of $50 \mathrm{deg} / \mathrm{sec}$
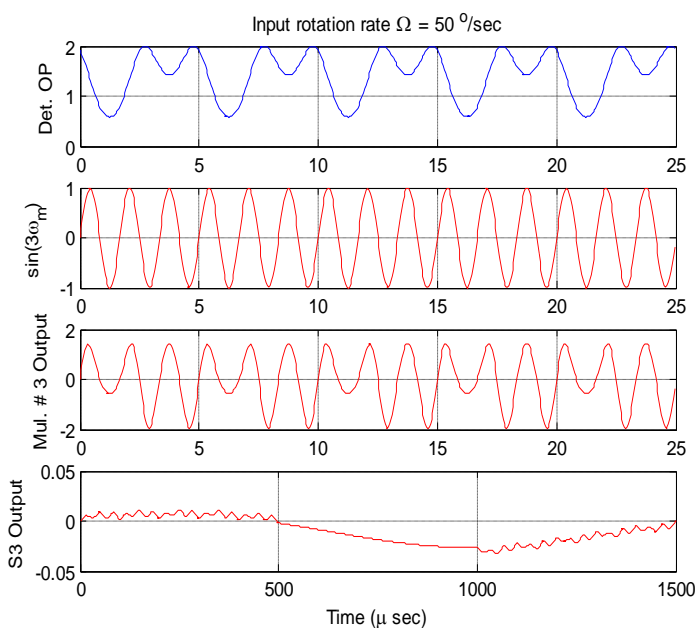

Graph 8.Graphical representation low pass filtered S3 for rotation rate of $50 \mathrm{deg} / \mathrm{sec}$
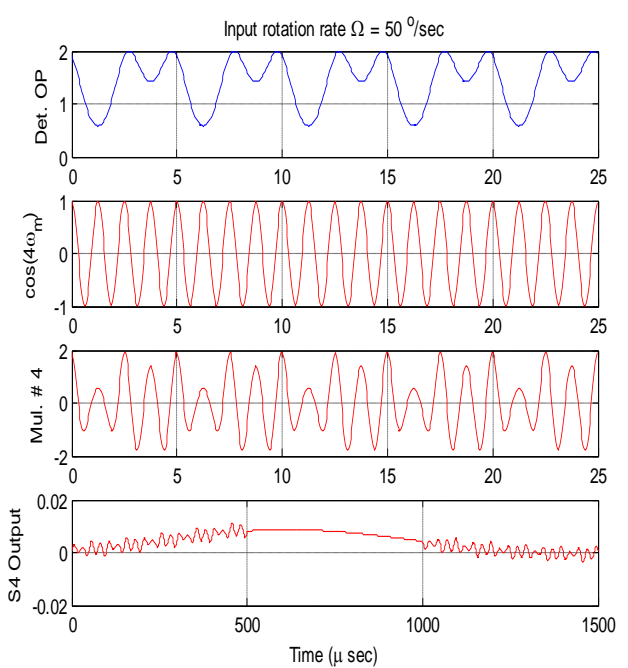

Graph 9. Graphical representation low pass filtered S4 for rotation rate of $50 \mathrm{deg} / \mathrm{sec}$
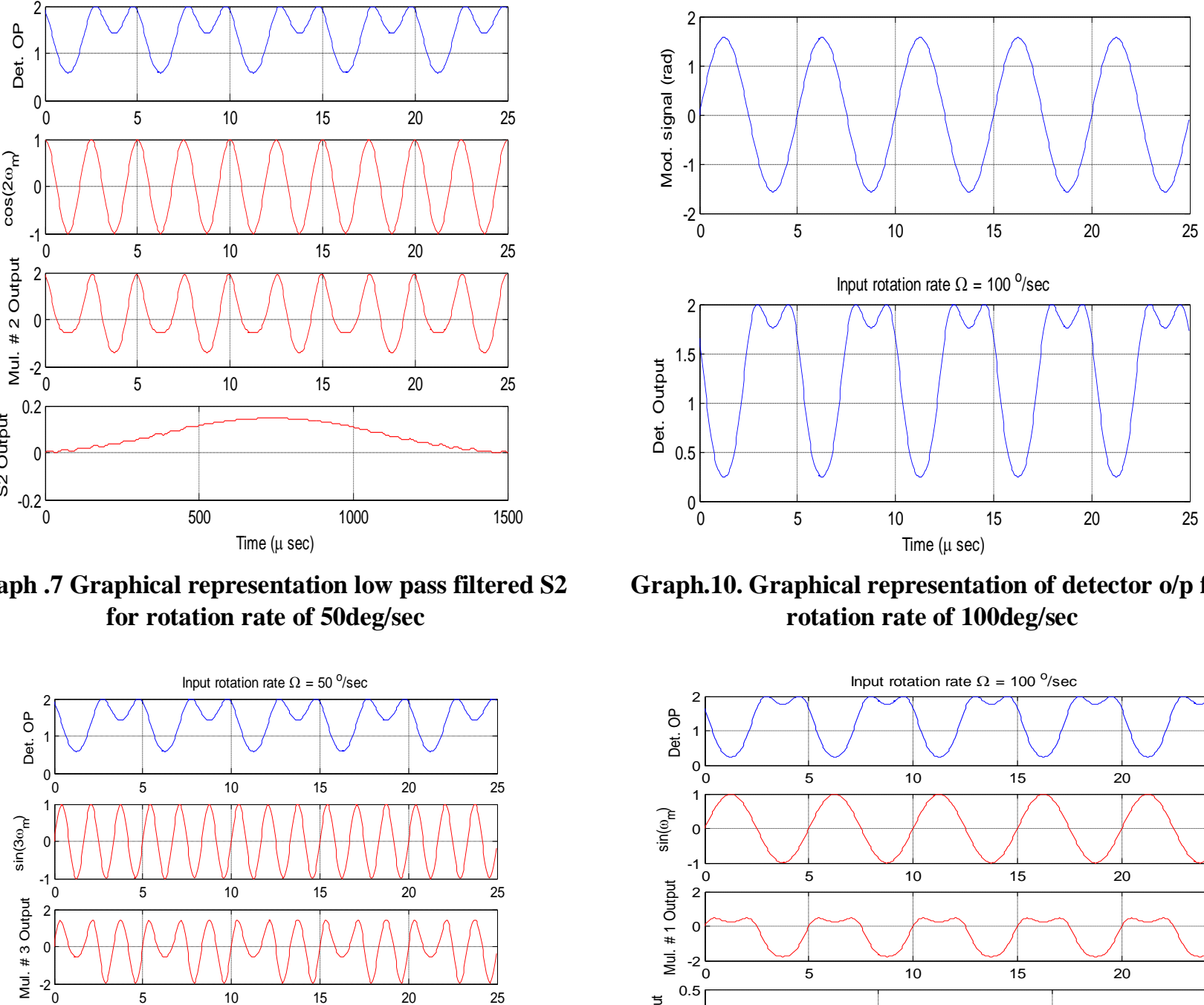

Graph.10. Graphical representation of detector o/p for rotation rate of $100 \mathrm{deg} / \mathrm{sec}$
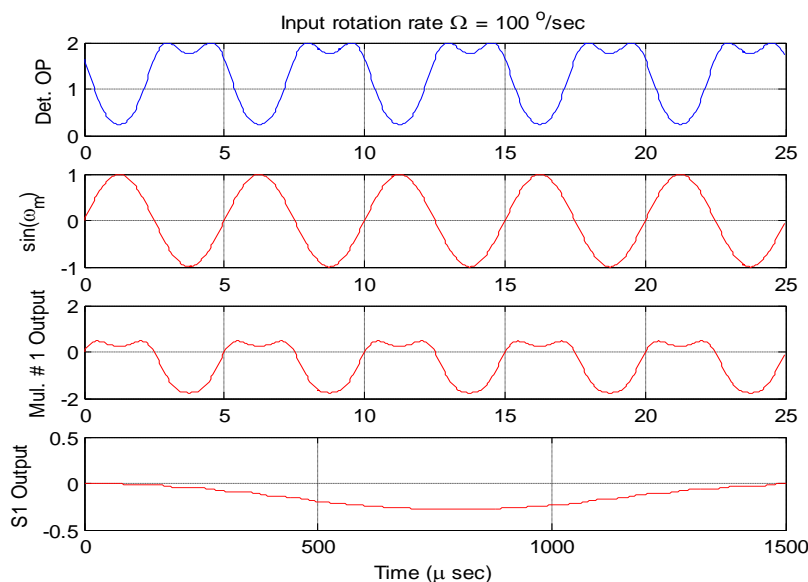

Graph.11. Graphical representation low pass filtered S1 for rotation rate of $100 \mathrm{deg} / \mathrm{sec}$
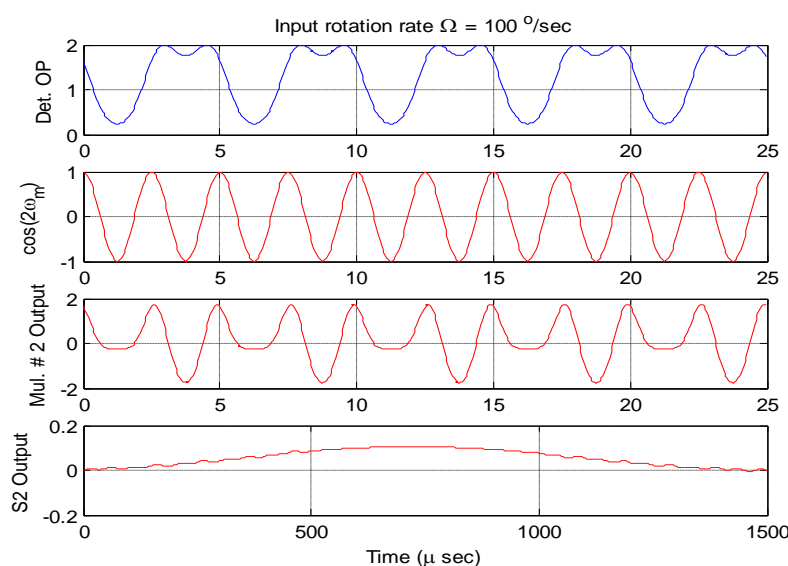

Graph 12.Graphical representation low pass filtered S2 for rotation rate of $100 \mathrm{deg} / \mathrm{sec}$ 

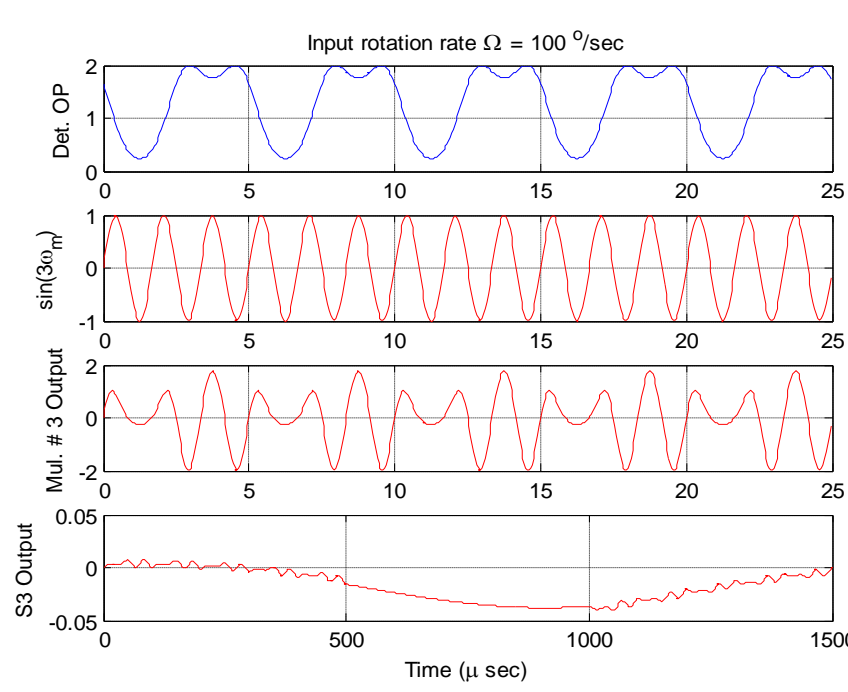

Graph.13. Graphical representation low pass filtered S3 for rotation rate of $100 \mathrm{deg} / \mathrm{sec}$
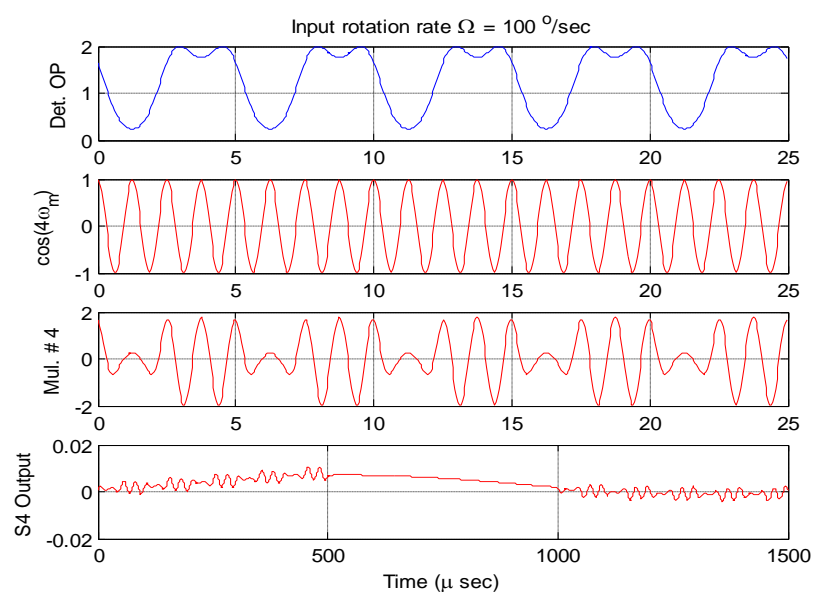

Graph.14. Graphical representation low pass filtered S4 for rotation rate of $10 \mathrm{deg} / \mathrm{sec}$

The rotation rate can be obtained by calculating

$\Delta \phi_{R}=\tan ^{-1}\left[-(S 1 / S 2)\left(J 2\left(\phi_{m}\right) / J 1\left(\phi_{m}\right)\right)\right]$

The graphical representation for rotation rate is shown below.

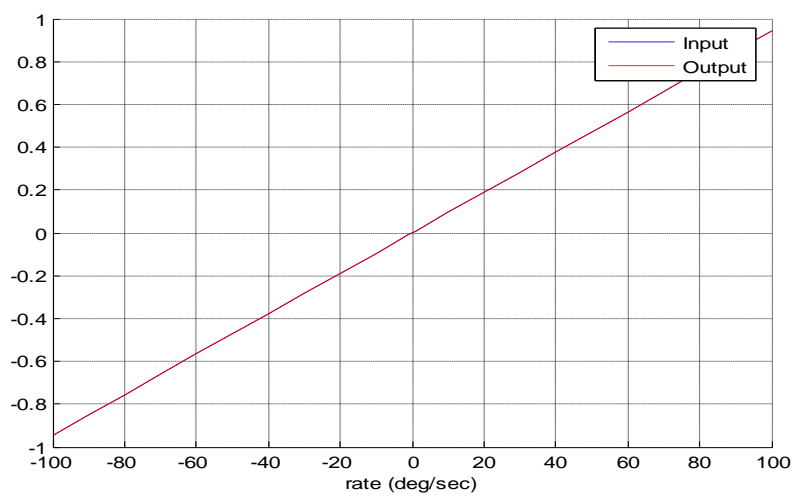

Graph 15.Graphical Representation of Rotation Rate

From the Graph (4-6) we conclude that, the rotation rate from the Equation (3) and Equation (6) will be equal and it satisfies the principle of sagnac effect,and it also satisfies the princile of linearit

\section{CONCLUSION}

Thus by performing the signal processing scheme for the basic sensor configuration, the limitations of it are reduced such as it becomes power independent ,it explains linearity and exact direction can be determined .As the final detector output equation does not contain any io components, even change in power does not effect the system hence it becomes power independent. By applying the biasing signal to the modulator, for low input values the output will be low and for high input values the output of the detector will be high. So, it also satisfies to explain the basic sensor principle and it gives the exact direction.

\section{REFERENCES}

1. Campbell, R. W. Tops and Gyroscopes. New York: Thomas Y. Crowell Company, 1959.

2. Langone, John. National Geographic's How Things Work: Everyday Technology Explained. Washington, DC: National Geographic Society, 1999 Culshaw, "Optical Fiber Sensing and Signal Processing", Peter Peregrinus, London 1984.

3. Dakin and B.Culshaw (Eds.), "Optical Fiber Sensors, Principles and Components", Vol. I \&II, Artech House, Boston 1988

4. Giallorenziet.al. "Optical Fiber Sensor Technology", IEEE J. Quantum Electronics QE-18, 1982, pp 626-665.

5. Batchellor and E. Edge, "Some Recent Advances in FiberOptic Sensors", Electron. \& Comm. Engg. J. Oct 1990 pp 175-184.

6. Kersey and A. Dandridge, "Applications of Fiber-Sensors", IEEE Trans. on Comp. Hybrids and Manufact. Technol. 13,1990 pp 137-143

7. Eric Udd (ed.), "Fiber Optic Sensors, An introduction for Engineers and Scientists", John Wiley \& Sons, Inc. New York, 1991.

8. Farhad Ansari (ed.), "Applications of Fiber Optic Sensors in Engineering Mechanics", American Society of Civil Engineers, New York 1993

9. Ezekiel and H.J. Arditty, "Fiber Optic Rotation Sensors", selected papers on Fiber Optic Gyroscopes, R.B. Smith (ed.), SPIE Press, 1989.

10. W.Su, J.A.Gilbert, C.Katsinis, "A photoelasticfiberoptic strain gage", Experimental Mechanics, March 1995, p.71-76.

11. P.A.Askins, G.M.Williams, E.J.Friebele, "Steppedwavelength optical-fiber Bragg grating arrays fabricated in line on a draw tower" , Optics Letters v 19 n 2 Jan 15 1994. p 147-149

12. Brooke's et.al, "Time Domain Addressing of Remote FiberOptic Interferometric Sensor Arrays", J. Lightwave Technol. LT-5, 1987 pp 1014-1023.

13. L.K.Melle, R.M. Measures, "Practical Fiber-Optic Bragg Grating Strain Gauge System", Appl. Optics, Vol. 32, No. 19, July 1993 pp 3601-3609.

14. Nishihara, M. Haruna , T. Suhaya, "Optical Integrated Circuits", Mc-Graw Hill, New York, 1989.

15. L.D.Hutcheson (Ed.), "Integrated Optical Circuits and Components", Marcel Dekkar Inc., 1987. 